CCNY-HEP-01/03

\title{
Chern-Simons matrix model: coherent states and relation to Laughlin wavefunctions
}

\author{
Dimitra Karabali ${ }^{a, c}$ and B. Sakita ${ }^{b, c}$ 円 \\ a Department of Physics and Astronomy, Lehman College of the CUNY \\ Bronx, NY 10468 \\ ${ }^{b}$ Physics Department, City College of the CUNY \\ New York, NY 10031 \\ c The Graduate School and University Center, CUNY \\ New York, NY 10016
}

\begin{abstract}
Using a coherent state representation we derive many-body probability distributions and wavefunctions for the Chern-Simons matrix model proposed by Polychronakos and compare them to the Laughlin ones. We analyze two different coherent state representations, corresponding to different choices for electron coordinate bases. In both cases we find that the resulting probability distributions do not quite agree with the Laughlin ones. There is agreement on the long distance behavior, but the short distance behavior is different.
\end{abstract}

\footnotetext{
${ }^{1}$ e-mail addresses: karabali@alpha.lehman.cuny.edu, sakita@scisun.sci.ccny.cuny.edu
} 


\section{Introduction}

There has recently appeared an interesting connection between quantum Hall effect and noncommutative field theory. In particular Susskind proposed in [1], that the Laughlin states at filling fractions $\nu=\frac{1}{2 p+1}$ for a system of an infinite number of electrons confined in the lowest Landau level (LLL) can be described by a noncommutative $U(1)$ Chern-Simons theory. The fields of this theory are infinite matrices which act on an infinite Hilbert space, appropriate to account for an infinite number of electrons. In the same spirit, Polychronakos, later, proposed a finite matrix model [2], a regularized version of this noncommutative Chern-Simons theory in an effort to describe finite systems, of limited spatial extent with a finite number of electrons. This matrix model was shown to reproduce the basic features of the quantum Hall droplets and their corresponding excitations [5], such as boundary and quasihole excitations at filling fraction $\nu=\frac{1}{2 p+1}$.

In a subsequent paper [3], Hellerman and Raamsdonk, trying to make the connection between quantum Hall effect and the noncommutative matrix model more transparent, analyzed the states of the theory and concluded that the states of the matrix model are in oneto-one correspondence with the Laughlin states describing QHE at filling fraction $\nu=\frac{1}{2 p+1}$. Similar arguments were put forward for the excited states. Although this is an interesting observation, the existence of a one-to-one mapping between states is not enough to prove the equivalence of the two theories.

Since the mapping in [3] is somewhat formal at the level of states, in this paper we try to go one step further and compare the two theories at the level of the wavefunctions. This requires a notion of coordinates, which is introduced via a coherent state representation for the states of the matrix model. We will present two different ways of arriving at a coherent state representation, which are not equivalent in general. In the special case of $\nu=1$ ( $\theta=0$ ), it turns out that both coherent state representations of the corresponding matrix model reproduce the $\nu=1$ Laughlin wavefunction. For $\nu=\frac{1}{2 p+1}$ though, the two ways produce different probability distributions and neither agrees with the Laughlin one. The probability distributions emerging from the different coherent state representations have a long distance behavior similar to the corresponding Laughlin one, but quite a different short distance behavior.

This paper is organized as follows. In section 2 we give a brief discussion of fermions in the lowest Landau level and the Laughlin wavefunctions. In section 3 we briefly review the Chern-Simons finite matrix model proposed by Polychronakos. In section 4 we present two different approaches towards a coherent state representation of the matrix states. In section 5 we comment on our results.

\section{Fermions in the lowest Landau level}

It is well known by now that the two-dimensional configuration space of charged particles in the LLL is equivalent to a one-dimensional phase space and therefore noncommutative 
[6, 77. To see this let us consider a charged particle in the presence of a strong uniform magnetic field. The Lagrangian is given by

$$
L=\frac{m}{2} \dot{\vec{x}}^{2}+\vec{A} \cdot \dot{\vec{x}}-V\left(x_{1}, x_{2}\right)
$$

where $V\left(x_{1}, x_{2}\right)$ is an abritrary confining potential. For convenience we shall consider $A_{0}$ to be a harmonic oscillator potential of the form

$$
V=\frac{1}{2} \omega \vec{x}^{2}
$$

The energy eigestates of this system lie on Landau levels and at the limit $m \rightarrow 0$ (or equivalently strong $B$ ) the system is confined to the LLL. In this case the kinetic energy term in (1) is negligible. We further use the radial gauge $A_{i}=\frac{1}{2} B \epsilon_{i j} x_{j}$. The canonical momentum of $x_{1}$ is then $p_{1}=B x_{2}$ and as a result

$$
\left[x_{1}, x_{2}\right]=\left[x_{1}, \frac{p_{1}}{B}\right]=\frac{i}{B}
$$

In the absence of a confining potential all the eigenstates at each Landau level are degenerate. The confining potential lifts the degeneracy. In the lowest Landau level the Hamiltonian is

$$
H=\frac{\omega}{B}\left(a^{\dagger} a+\frac{1}{2}\right)
$$

where $a=\sqrt{\frac{B}{2}}\left(x_{1}+i x_{2}\right)$. The single particle eigenstates are

$$
|n\rangle=\frac{1}{\sqrt{n !}} a^{\dagger n}|0\rangle
$$

The corresponding normalized wavefunctions are

$$
\Psi_{n}=\sqrt{\frac{B}{2 \pi n !}} \bar{z}^{n} e^{-|z|^{2} / 2}
$$

where $z=\sqrt{\frac{B}{2}}\left(x_{1}+i x_{2}\right)$. These can be thought of as the coherent state representation of (5).

Consider now the case of $N$ fermions. Since each state can be occupied by at most one fermion, the presence of the confining potential selects a unique ground state which is the minimum angular momentum state. This is the $\nu=1$ ground state wavefunction

$$
\Psi_{1}\left(\vec{x}_{1}, \ldots, \vec{x}_{N}\right)=\prod_{i<j}\left(\bar{z}_{i}-\bar{z}_{j}\right) e^{-\frac{1}{2} \sum_{i=1}^{N}\left|z_{i}\right|^{2}}
$$

This corresponds to an incompressible circular droplet configuration of uniform density $\rho=$ $\frac{B}{2 \pi}$. This incompressibility is crucial in explaining the experimentally observed gap for $\nu=1$. 
The existence of a similar gap is less obvious in the case of noninteger filling fractions, where the LLL is only partially filled. In this case, it is believed that the repulsive Coulomb interactions among electrons are important in generating strong correlations which eventually produce a new ground state with a gap. Laughlin proposed that such a state, in the case $\nu=\frac{1}{2 p+1}$, is described by a wavefunction 8

$$
\Psi_{2 p+1}\left(\vec{x}_{1}, \ldots, \vec{x}_{N}\right)=\prod_{i<j}^{N}\left(\bar{z}_{i}-\bar{z}_{j}\right)^{2 p+1} e^{-\frac{1}{2} \sum_{i=1}^{N}\left|z_{i}\right|^{2}}
$$

Using the connection to the one-component, two-dimensional plasma, Laughlin showed that

this corresponds to an incompressible droplet of density $\rho=\frac{B}{2 \pi(2 p+1)}$. Although not exact eigenfunctions, (8) are quite close to the true solutions, at least numerically. They vanish quite rapidly if any two particles approach each other, and this helps minimize the expectation value of the Coulomb energy. Their success lies very much on their short distance behavior.

Our aim is to develop an appropriate coherent state representation for the states of the corresponding matrix model and compare them directly to the Laughlin wavefunctions. Before we explain this in more detail, we shall give a brief review of the matrix Chern-Simons model proposed by Polychronakos.

\section{Chern-Simons matrix model}

The action describing the matrix Chern-Simons model is given by [2]

$$
S=\int d t \frac{B}{2} \operatorname{Tr}\left\{\epsilon_{a b}\left(\dot{X}_{a}+i\left[A_{0}, X_{a}\right]\right) X_{b}+2 \theta A_{0}-\omega X_{a}^{2}\right\}+\Psi^{\dagger}\left(i \dot{\Psi}-A_{0} \Psi\right)
$$

where $X_{a}, a=1,2$ are $N \times N$ matrices and $\Psi$ is a complex $N$-vector that transforms in the fundamental of the gauge group $U(N)$,

$$
X_{a} \rightarrow U X_{a} U^{-1}, \quad \Psi \rightarrow U \Psi
$$

The $A_{0}$ equation of motion implies the constraint

$$
G \equiv-i B\left[X_{1}, X_{2}\right]+\Psi \Psi^{\dagger}-B \theta=0
$$

The trace of this equation gives

$$
\Psi^{\dagger} \Psi=N B \theta
$$

It is interesting to note that in the absence of $\Psi$ 's, the parameter $\theta$ has to be zero. In this case the action (9) is that of a one-dimensional hermitian matrix model in a harmonic potential, which is known to be equivalent to $N$ one-dimensional fermions in a confining potential [9]. 
Upon quantization the matrix elements of $X_{a}$ and the components of $\Psi$ become operators, obeying the following commutation relations

$$
\begin{aligned}
{\left[\Psi_{i}, \Psi_{j}^{\dagger}\right] } & =\delta_{i j} \\
{\left[\left(X_{1}\right)_{i j},\left(X_{2}\right)_{k l}\right] } & =\frac{i}{B} \delta_{i l} \delta_{j k}
\end{aligned}
$$

The Hamiltonian is

$$
H=\omega\left(\frac{N^{2}}{2}+\sum A_{i j}^{\dagger} A_{j i}\right)
$$

where $A=\sqrt{\frac{B}{2}}\left(X_{1}+i X_{2}\right)$. The system contains $N(N+1)$ oscillators coupled by the constraint (11). As explained in [2], upon quantization, the operator $G$ becomes the generator of unitary rotations of both $X_{a}$ and $\Psi$. The trace part (12) demands that $N B \theta$ being the number operator for $\Psi$ 's is quantized to an integer. The traceless part of the constraint demands the physical states to be singlets of $S U(N)$.

Since the $A_{i j}^{\dagger}$ transform in the adjoint and the $\Psi_{i}^{\dagger}$ transform in the fundamental representation of $S U(N)$, a purely group theoretical argument implies that a physical state being a singlet has to contain $N n \Psi^{\dagger}$ 's, where $n$ is an integer. This leads to the quantization of $B \theta=k$. We shall see later that in identifying this matrix model with a fermionic system, $k$ has to be an even integer.

Explicit expressions for the states were written down in [3]. (Essentially equivalent results were also obtained by Polychronakos [⿴囗十).) The ground state being an $S U(N)$ singlet with the lowest number of $A^{\dagger}$ 's is of the form

$$
|\Psi\rangle=\left[\epsilon^{i_{1} \ldots i_{N}} \Psi_{i_{1}}^{\dagger}\left(\Psi^{\dagger} A^{\dagger}\right)_{i_{2} \ldots} \ldots\left(\Psi^{\dagger} A^{\dagger N-1}\right)_{i_{N}}\right]^{k}|0\rangle
$$

where $|0\rangle$ is annihilated by $A$ 's and $\Psi$ 's, while the excited states can be written as

$$
\left|\Psi_{\text {exc }}\right\rangle=\prod_{i=1}^{N-1}\left(\operatorname{Tr} A^{\dagger i}\right)^{c_{i}}\left[\epsilon^{i_{1} \ldots i_{N}} \Psi_{i_{1}}^{\dagger}\left(\Psi^{\dagger} A^{\dagger}\right)_{i_{2}} \ldots\left(\Psi^{\dagger} A^{\dagger N-1}\right)_{i_{N}}\right]^{k}|0\rangle
$$

\section{Coherent state representations}

We now present a coherent state representation for the matrix states (15, 16). In doing this one has to make a choice of coordinates, which eventually parametrize the phase space of the underlying one-dimensional system.

From the matrix model point of view, a natural choice would be to diagonalize $A$ and interpret its eigenvalues as phase space coordinates of particles. We refer to this as the $A$-representation. This is essentially a generalized complex random matrix model. 
Another choice would be to diagonalize $X_{1}$ and interpret its eigenvalues as one-dimensional coordinates. This is the $X$-representation. In this, the elements of $X_{2}$ are canonically conjugate to $X_{1}$. Once the $X$-representation of the matrix states (15, 16) is derived, we can then use the usual coherent state representation to express the wavefunctions in terms of phase space coordinates.

Clearly in the two cases, $A$ - and $X$-representation, the notion of phase space coordinates is different; as a result we derive different expressions for the corresponding wavefunctions.

Below we present in detail the two representations and compare the results to the Laughlin wavefunctions.

$\underline{A-\text { representation }}$

We define the coherent state $|Z, \phi\rangle$ such that

$$
\begin{aligned}
A_{m n}|Z, \phi\rangle & =Z_{m n}|Z, \phi\rangle \\
\Psi_{n}|Z, \phi\rangle & =\phi_{n}|Z, \phi\rangle
\end{aligned}
$$

where $Z$ is a complex $N \times N$ matrix and $\phi$ is a complex vector. Let us consider the matrix ground state of the form

$$
|2 p+1\rangle=\left[\epsilon^{i_{1} \ldots i_{N}} \Psi_{i_{1}}^{\dagger}\left(\Psi^{\dagger} A^{\dagger}\right)_{i_{2} \ldots}\left(\Psi^{\dagger} A^{\dagger N-1}\right)_{i_{N}}\right]^{2 p}|0\rangle
$$

and reexpress its scalar product in terms of the coherent state wavefunctions using the completeness relation

$$
\langle 2 p+1 \mid 2 p+1\rangle=\int \prod_{i, j, l}^{N} d Z_{i j} d Z_{i j}^{*} d \phi_{l} d \bar{\phi}_{l}\langle 2 p+1 \mid Z, \phi\rangle\langle Z, \phi \mid 2 p+1\rangle
$$

Before we talk about the general case, it is interesting to demonstrate how the coherent state representation works for $p=0$. The scalar product (19) can be written as,

$$
\langle 1 \mid 1\rangle=\int \prod_{i, j, l}^{N} d Z_{i j} d Z_{i j}^{*} e^{-T r Z^{\dagger} Z} \int d \phi_{l} d \bar{\phi}_{l} e^{-\bar{\phi} \phi}
$$

Since the $\phi$ integration is trivial, eq. (20) defines essentially a complex random matrix model with probability distribution $e^{-\operatorname{Tr} Z^{\dagger} Z}$. A detailed analysis of this model has been given in [10].

$Z$ is a complex matrix that can be diagonalized as, (nondiagonalizable matrices form a set of measure zero)

$$
Z=X E X^{-1}
$$

where $E$ is diagonal with $E_{i i}=z_{i}$. Integration over the nondiagonal part of $Z$ in (20) gives the following result 10

$$
\langle 1 \mid 1\rangle=\int \prod_{i=1}^{N} d z_{i} d \bar{z}_{i} e^{-\left|z_{i}\right|^{2}} \prod_{i<j}\left|z_{i}-z_{j}\right|^{2}
$$


up to normalization factors. One can immediatelly recognize the integrand of (22) as the probability distribution corresponding to the $\nu=1$ Laughlin wavefunction. In Arepresentation we have identified the phase space coordinates of the fermions with the eigenvalues of the matrix $Z$ in (17).

We would like now to extend this approach in the case $p \neq 0$ in the presence of the extra $\Psi$ degrees of freedom. This can be viewed as a generalized complex random matrix model. (An attempt for a random matrix formulation of the Laughlin theory of the fractional QHE has been proposed by Callaway [11].) As shown in [10 any complex regular $N \times N$ matrix $X$ can be expressed in one and only one way as

$$
X=U Y V
$$

where $U$ is a unitary matrix, $Y$ is a triangular matrix with $Y_{i j}=0$ for $i>j$ and $Y_{i i}=1$ and $V$ is a diagonal matrix with real positive diagonal elements. Using this particular parametrization we find

$$
\begin{aligned}
& <2 p+1 \mid Z, \phi>=\left[\epsilon^{i_{1} \ldots i_{N}} \phi_{i_{1}}(Z \phi)_{i_{2}}\left(Z^{2} \phi\right)_{i_{2}} \ldots\left(Z^{N-1} \phi\right)_{i_{N}}\right]^{2 p} e^{-\frac{1}{2} \operatorname{Tr} Z^{\dagger} Z} e^{-\frac{1}{2} \bar{\phi} \phi} \\
& =\left[\epsilon^{i_{1} \ldots i_{N}} \phi_{i_{1}}\left(U Y E Y^{-1} U^{-1} \phi\right)_{i_{2}}\left(U Y E^{2} Y^{-1} U^{-1} \phi\right)_{i_{2} \ldots} \ldots\left(U Y E^{N-1} Y^{-1} U^{-1} \phi\right)_{i_{N}}\right]^{2 p} e^{-\frac{1}{2} \operatorname{Tr} Z^{\dagger} Z} e^{-\frac{1}{2} \bar{\phi} \phi} \\
& =[\operatorname{det}(U Y)]^{2 p} \prod_{i<j}\left(z_{i}-z_{j}\right)^{2 p} \prod_{i=1}^{N} \xi_{i}^{2 p} e^{-\frac{1}{2} \operatorname{Tr} Z^{\dagger} Z} e^{-\frac{1}{2} \bar{\phi} \phi}
\end{aligned}
$$

where $\xi=(U Y)^{-1} \phi$.

Further using (21) and (23) we have

$$
\operatorname{Tr} Z^{\dagger} Z=\operatorname{Tr}\left(\bar{E} H E H^{-1}\right)
$$

where $H=Y^{\dagger} Y$ and $\operatorname{det} \mathrm{H}=1(\operatorname{det} \mathrm{Y}=1)$.

In performing the integration over $\phi$ 's it is convenient to change variables from $\phi$ to $\xi$. Since $U$ is unitary and $\operatorname{det} Y=1$

$$
\prod_{l=1}^{N} d \bar{\phi}_{l} d \phi_{l} e^{-\bar{\phi} \phi}=\prod_{l=1}^{N} d \bar{\xi}_{l} d \xi_{l} e^{-\bar{\xi} H \xi}
$$

Further following [10], one can show that

$$
\begin{aligned}
d Z_{i j} d Z_{i j}^{*} & =\prod_{i} d z_{i} d \bar{z}_{i} \prod_{i<j}\left|z_{i}-z_{j}\right|^{4} \prod_{i \neq j} d H_{i j} \\
& \equiv d \mu(z, H) \prod_{i<j}\left|z_{i}-z_{j}\right|^{4}
\end{aligned}
$$

upto normalization factors. 
Putting everything together we find

$$
\langle 2 p+1 \mid 2 p+1\rangle=\int d \mu(z, H) \prod_{i<j}\left|z_{i}-z_{j}\right|^{4 p+4} e^{-\operatorname{Tr}\left(\bar{E} H E H^{-1}\right)} d \bar{\xi}_{i} d \xi_{i} \prod_{i=1}^{N}\left(\bar{\xi}_{i} \xi_{i}\right)^{2 p} e^{-\xi^{\dagger} H \xi}
$$

Integration over $\xi$ and $H$ would produce a quantity that depends only on $z_{i}$, $\bar{z}_{i}$. It is clear from the above expression that in the absence of the $\xi$ integration, the integration over the nondiagonal elements of $Z$ would produce a probability distribution similar to the corresponding Laughlin one. Since however $\xi$ 's couple to $H$ and $H$ in turn couples to $z_{i}$ and $\bar{z}_{i}$, this integration will not necessarily produce a probability distribution which agrees with the Laughlin one. The integration over $\xi$ and $H$ is a lot more involved now and it is hard to extract a closed expression for arbitrary $N$. However, as we shall see one can find general features of the probability distribution, which do not agree with what one would expect from the Laughlin distribution.

We first perform the $\xi$-integration by introducing a source term as follows

$$
\begin{aligned}
& \int d \xi_{i} d \bar{\xi}_{i} \prod_{i=1}^{N}\left(\xi_{i} \bar{\xi}_{i}\right)^{2 p} e^{-\bar{\xi} H \xi} \\
= & \left.\prod_{i=1}^{N}\left(\frac{\partial}{\partial J_{i}} \frac{\partial}{\partial \bar{J}_{i}}\right)^{2 p} \int d \xi_{i} d \bar{\xi}_{i} e^{-\bar{\xi} H \xi+\bar{J} \xi+J \bar{\xi}}\right|_{J, \bar{J}=0} \\
= & \left.\prod_{i=1}^{N}\left(\frac{\partial}{\partial J_{i}} \frac{\partial}{\partial \bar{J}_{i}}\right)^{2 p} e^{\bar{J} H^{-1} J}\right|_{J, \bar{J}=0}
\end{aligned}
$$

Eq. (28) can now be written as

$$
\langle 2 p+1 \mid 2 p+1\rangle=\left.\int \prod_{i} d z_{i} d \bar{z}_{i} \prod_{i<j}\left|z_{i}-z_{j}\right|^{4 p+4} \prod_{i=1}^{N}\left(\frac{\partial}{\partial J_{i}} \frac{\partial}{\partial \bar{J}_{i}}\right)^{2 p} \int \prod_{i \neq j} d H_{i j} e^{-\operatorname{Tr}\left(\bar{E} H E H^{-1}\right)+\bar{J} H^{-1} J}\right|_{J, \bar{J}=0}
$$

We are now left with the $H$ integration

$$
I=\int \prod_{1 \leq i \neq j \leq N} d H_{i j} e^{-\operatorname{Tr}\left(\bar{E} H E H^{-1}\right)} e^{\bar{J} H^{-1} J}
$$

To do this we follow an iterative procedure as in [10]. At each step of the iteration we integrate over the variables of the last row and column of $H$ and thus decrease by one the size of the matrix. In the absence of the source terms, the structure of the reduced matrix remains the same and this produces a simple recursion formula, which eventually leads to (22). This is not quite the case when there are source terms, as a result there is no simple recursion formula.

The iteration procedure is defined as follows: Let $H^{\prime}, E^{\prime}$, etc., be the relevant matrices of order $n$ and $H, E$, be those obtained from $H^{\prime}, E^{\prime}$ by removing the last row and last column. 
Greek (Latin) indices run from 1 to $n(n-1)$. Let $\Delta_{\alpha \beta}^{\prime}$ be the cofactor of $H_{\alpha \beta}^{\prime}$ in $H^{\prime}$ and $\Delta_{i j}$, the cofactor of $H_{i j}$ in $H$. Let $g_{i}=H_{i n}^{\prime}$. Because $\operatorname{det} H^{\prime}=\operatorname{det} H=1$, the following relations are true [10],

$$
\begin{aligned}
& \Delta_{\alpha \beta}^{\prime}=H_{\beta \alpha}^{\prime-1}, \quad \Delta_{i j}=H_{j i}^{-1} \\
& H_{n n}^{\prime}=1+\sum_{i, j} g_{i}^{*} g_{j} \Delta_{j i} \\
& \Delta_{i n}^{\prime}=-\sum_{l} \Delta_{i l} g_{l}^{*} \\
& \Delta_{i j}^{\prime}=H_{n n}^{\prime} \Delta_{i j}-\sum_{l, k} g_{k}^{*} g_{l} \Delta_{i j}^{l k} \\
& \Delta_{i j}^{l k}=\Delta_{i j} \Delta_{l k}-\Delta_{i k} \Delta_{l j}
\end{aligned}
$$

Let

$$
\Phi_{n}^{J}=\operatorname{Tr}\left(\bar{E}^{\prime} H^{\prime} E^{\prime} H^{\prime}-1\right)-\bar{J}^{\prime} H^{\prime}-1 J^{\prime} \equiv \Phi_{n}^{0}-\bar{J}^{\prime} H^{\prime}-1 J^{\prime}
$$

Using (32) we find

$$
\begin{aligned}
\Phi_{N}^{J}= & \left|z_{N}\right|^{2}+\Phi_{N-1}^{J}-J_{N} \bar{J}_{N}+\left\langle g\left|H^{-1}\left(\bar{E}-\bar{z}_{N}\right) H\left(E-z_{N}\right) H^{-1}\right| g\right\rangle \\
& -\left\langle g\left|H^{-1} J \bar{J} H^{-1}\right| g\right\rangle+g_{l}^{*}\left(H^{-1} J\right)_{l} \bar{J}_{N}+J_{N}\left(\bar{J} H^{-1}\right)_{l} g_{l}
\end{aligned}
$$

Integration over $g$ 's produces the following result

$$
I \sim \int \prod_{1 \leq i \neq j \leq N-1} d H_{i j} e^{-\left|z_{N}\right|^{2}} e^{-\Phi_{N-1}^{J}} e^{J_{N} \bar{J}_{N}} \frac{e^{\left(\sum \bar{J}_{i} X_{i j}^{-1} J_{j}\right) J_{N} \bar{J}_{N}}}{\operatorname{det}\left(X_{i j}-J_{i} \bar{J}_{j}\right)}
$$

where

$$
X_{i j}=\left(H^{-1}\left(\bar{E}-\bar{z}_{N}\right) H\left(E-z_{N}\right) H^{-1}\right)_{i j}-J_{i} \bar{J}_{j}=X_{i j}^{0}-J_{i} \bar{J}_{j}
$$

The expression (35) can be further simplified using the following relations

$$
\bar{J}_{i}\left(X^{0}-J \bar{J}\right)_{i j}^{-1} J_{j}=\bar{J}_{i}\left(\left(X^{0}\right)^{-1}+\left(X^{0}\right)^{-1} J \bar{J}\left(X^{0}\right)^{-1}+\ldots\right)_{i j} J_{j}=\frac{\bar{J}_{i}\left(X^{0}\right)_{i j}^{-1} J_{j}}{1-\bar{J}_{i}\left(X^{0}\right)_{i j}^{-1} J_{j}}
$$

Further

$$
\begin{aligned}
& \operatorname{det}\left(X^{0}-J \bar{J}\right)=e^{\operatorname{Tr} \log \left(X^{0}-J \bar{J}\right)}=e^{\operatorname{Tr}\left[\log X^{0}+\log \left(1-\left(X^{0}\right)^{-1} J \bar{J}\right)\right]} \\
& =\operatorname{det}\left(X^{0}\right) e^{\log \bar{J}\left(X^{0}\right)^{-1} J}=\prod_{i<N}\left|z_{i}-z_{N}\right|^{2} \sum_{1 \leq l, m \leq N-1} \bar{J}_{l}\left(X^{0}\right)_{l m}^{-1} J_{m}
\end{aligned}
$$

where we have used that

$$
\operatorname{det} X^{0}=\operatorname{det}\left(H^{-1}\left(\bar{E}-\bar{z}_{N}\right) H\left(E-z_{N}\right) H^{-1}\right)=\prod_{i<N}\left|z_{i}-z_{N}\right|^{2}
$$


Substituting (37, 38) in (35) we find

$$
I \sim \frac{e^{-\left|z_{N}\right|^{2}}}{\prod_{i<N}\left|z_{i}-z_{N}\right|^{2}} \int \prod_{1 \leq i \neq j \leq N-1} d H_{i j} \frac{e^{-\Phi_{N-1}^{J}}}{(1-M)} e^{\frac{J_{N} \bar{J}_{N}}{1-M}}
$$

where

$$
M=\sum_{1 \leq i, j \leq N-1} \bar{J}_{i}\left(X^{0}\right)_{i j}^{-1} J_{j}=\sum_{1 \leq i, j \leq N-1} \frac{\bar{J}_{i}}{\left(z_{i}-z_{N}\right)} H_{i j}^{-1} \frac{J_{j}}{\left(\bar{z}_{j}-\bar{z}_{N}\right)}
$$

Already this result can be used to evaluate (28) for the simple case of a $2 \times 2$ matrix model. Although this is a rather trivial case, it is worth presenting it, since it highlights properties of the probability distribution which are not in agreement with the Laughlin one.

To simplify the calculation let us further choose $p=1$. For the $N=2$ case after the first iteration we find from (40)

$$
I \sim \frac{e^{-\left|z_{1}\right|^{2}} e^{-\left|z_{2}\right|^{2}}}{\left|z_{1}-z_{2}\right|^{2}} \frac{e^{J_{1} \bar{J}_{1}}}{1-M} e^{\frac{J_{2} \bar{J}_{2}}{1-M}}
$$

where $M=1-\frac{J_{1} \bar{J}_{1}}{\left|z_{1}-z_{2}\right|^{2}}$. The functional diferentiation with respect to $J$ 's, eq. (29), gives

$$
\begin{aligned}
& \left.\left(\frac{\partial}{\partial J_{1}} \frac{\partial}{\partial \bar{J}_{1}}\right)^{2}\left(\frac{\partial}{\partial J_{2}} \frac{\partial}{\partial \bar{J}_{2}}\right)^{2} I\right|_{J, \bar{J}=0} \\
& =\left.2 \frac{e^{-\left|z_{1}\right|^{2}} e^{-\left|z_{2}\right|^{2}}}{\left|z_{1}-z_{2}\right|^{2}}\left(\frac{\partial}{\partial J_{1}} \frac{\partial}{\partial \bar{J}_{1}}\right)^{2} \frac{e^{J_{1} \bar{J}_{1}}}{\left(1-\frac{J_{1} \bar{J}_{1}}{\left|z_{1}-z_{2}\right|^{2}}\right)^{3}}\right|_{J_{1}, \bar{J}_{1}=0} \\
& =\frac{e^{-\left|z_{1}\right|^{2}} e^{-\left|z_{2}\right|^{2}}}{\left|z_{1}-z_{2}\right|^{2}}\left[4+\frac{24}{\left|z_{1}-z_{2}\right|^{2}}+\frac{48}{\left|z_{1}-z_{2}\right|^{4}}\right]
\end{aligned}
$$

Using (28), (29) and (43) we find

$$
\langle 3 \mid 3\rangle \sim \int d z_{1} d \bar{z}_{1} d z_{2} d \bar{z}_{2} e^{-\left|z_{1}\right|^{2}-\left|z_{2}\right|^{2}}\left|z_{1}-z_{2}\right|^{6}\left[1+\frac{6}{\left|z_{1}-z_{2}\right|^{2}}+\frac{12}{\left|z_{1}-z_{2}\right|^{4}}\right]
$$

The first term corresponds to the probability distribution for the $\nu=1 / 3$ ground state Laughlin wavefunction. There are extra terms though, which are dominant at short distances as $z_{1} \rightarrow z_{2}$. In this simple case we find that the distribution emerging from the matrix model has a long distance behavior similar to the corresponding Laughlin one, but its short distance behavior is quite different. We shall now argue that this behavior prevails for any $N$.

It is clear from (40) that the first step of the iteration produces an expression which is not quite similar to the original one if $J^{\prime} s \neq 0$. As a result the integration over $H_{i n}^{\prime}$ at each subsequent step of the iteration becomes quite involved. Although it is very hard to derive an exact expression for $I$ as a function of $z_{i}$ 's, it is quite straightforward to explore its dependence on $\left|z_{N-1}-z_{N}\right|$. This is sufficient for example to get information about the 
short and long distance behavior of the probability distribution as $\left|z_{N-1}-z_{N}\right|<<1$ and $\left|z_{N-1}-z_{N}\right|>>1$ respectively.

The dependence of $I$ on $\left|z_{N-1}-z_{N}\right|$ comes first from the overall factor $1 / \prod_{i<N}\left|z_{i}-z_{N}\right|^{2}$ and second from the factor $M$, see eq. (40), (41). Expanding $M$ we find

$$
\begin{aligned}
M= & \frac{\bar{J}_{N-1} \bar{J}_{N-1}}{\left|z_{N-1}-z_{N}\right|^{2}}+\frac{\bar{J}_{N-1}}{\left(z_{N-1}-z_{N}\right)} \sum_{j<N-1} H_{N-1, j}^{-1} \frac{J_{j}}{\left(\bar{z}_{j}-\bar{z}_{N}\right)}+\sum_{i<N-1} \frac{\bar{J}_{i}}{\left(z_{i}-z_{N}\right)} H_{i, N-1}^{-1} \frac{J_{N-1}}{\left(\bar{z}_{N-1}-\bar{z}_{N}\right)} \\
& +\sum_{1 \leq i, j \leq N-2} \frac{\bar{J}_{i}}{\left(z_{i}-z_{N}\right)} H_{i j}^{-1} \frac{J_{j}}{\left(\bar{z}_{j}-\bar{z}_{N}\right)}
\end{aligned}
$$

It is clear now that in order to explicitly demonstrate the $\left|z_{N}-z_{N-1}\right|$ dependence we need to evaluate the functional derivatives with respect to $J_{N}$ and $J_{N-1}$, as in (30). We find

$$
\begin{aligned}
& \left.\prod_{i}\left(\frac{\partial}{\partial J_{i}} \frac{\partial}{\partial \bar{J}_{i}}\right)^{2} I\right|_{J, \bar{J}=0}=\frac{e^{-\sum_{i}\left|z_{i}\right|^{2}}}{\prod_{i<N}\left|z_{i}-z_{N}\right|^{2}}\left(A+\frac{B}{z_{N-1}-z_{N}}+\frac{B^{*}}{\bar{z}_{N-1}-\bar{z}_{N}}\right. \\
& +\frac{C}{\left|z_{N-1}-z_{N}\right|^{2}}+\frac{D}{\left(z_{N-1}-z_{N}\right)^{2}}+\frac{D^{*}}{\left(\bar{z}_{N-1}-\bar{z}_{N}\right)^{2}} \\
& \left.+\frac{1}{\left|z_{N-1}-z_{N}\right|^{2}}\left(\frac{E}{z_{N-1}-z_{N}}+\frac{\bar{E}}{\bar{z}_{N-1}-\bar{z}_{N}}\right)+\frac{F}{\left|z_{N-1}-z_{N}\right|^{4}}\right)
\end{aligned}
$$

where $A, B, C, D, E, F$ are in general functions of $z$ 's containing factors of the form $\left(z_{i}-z_{N}\right),\left(z_{i}-z_{N-1}\right),\left(z_{i}-z_{j}\right)$ and their complex conjugates, where $i \leq N-2$. Substituting this expression in (30) we find a probability distribution which is dominated by a term proportional to $\left|z_{N}-z_{N-1}\right|^{6}$ when $\left|z_{N}-z_{N-1}\right|>>1$, similar to the $\nu=1 / 3$ Laughlin distribution. However when $\left|z_{N}-z_{N-1}\right|<<1$ the matrix distribution is dominated by a term proportonal to $\left|z_{N}-z_{N-1}\right|^{2}$. The long and short distance behavior for any $N$ is the same as the one found in the simple $N=2$ case, eq.(44).

It is straightforward now to see that similar results can be derived for any $p \neq 0$. In particular the long distance behavior of the probability distribution is that of the $\nu=$ $\frac{1}{2 p+1}$ Laughlin distribution while the short distance behavior is that of a $\nu=1$ Laughlin distribution.

Further the probability distribution cannot be factorized as $\Psi^{*} \Psi$ where $\Psi$ is the corresponding many-particle wavefunction, which is both antisymmetric and holomorphic in $z$ 's. This indicates that the identification of the eigenvalues of the matrix $Z$ with the actual holomorphic coordinates of fermions may not be appropriate.

$\underline{X-\text { representation }}$

Going to an $X$-representation first we derive one-dimensional fermionic wavefunctions, where the coordinates have been identified with the eigenvalues of the matrix $X_{1}$, then transform the wavefunctions to the coherent state representation. 
We define the state $|X, \phi\rangle$ such that

$$
\hat{X}_{1}|X, \phi\rangle=X|X, \phi\rangle \quad \Psi|X, \phi\rangle=\phi|X, \phi\rangle
$$

Using (47) we find

$$
\langle 2 p+1 \mid X, \phi\rangle=\left[\epsilon^{i_{1} \ldots i_{N}} \phi_{i_{1}}(A \phi)_{i_{2} \ldots} \ldots\left(A^{N-1} \phi\right)_{i_{N}}\right]^{2 p}\langle 0 \mid X, \phi\rangle
$$

where

$$
\begin{aligned}
\langle 0 \mid X, \phi\rangle & =e^{-\operatorname{Tr} \frac{1}{2} B X^{2}} e^{-\frac{1}{2} \bar{\phi} \phi} \\
A_{i j} & =\sqrt{\frac{B}{2}}\left(X_{i j}-\frac{1}{B} \frac{\partial}{\partial X_{j i}}\right)
\end{aligned}
$$

Since (48) is completely antisymmetric in the $i_{n}$-indices, the differential operator $\frac{\partial}{\partial X_{j i}}$ produces a nonzero contribution only if it acts on the ground state wavefunction $\langle 0 \mid X, \phi\rangle$. We then find

$$
\langle 2 p+1 \mid X, \phi\rangle=(\sqrt{2 B})^{p N(N-1)}\left[\epsilon^{i_{1} \ldots i_{N}} \phi_{i_{1}}(X \phi)_{i_{2}} \ldots\left(X^{N-1} \phi\right)_{i_{N}}\right]^{2 p} e^{-\operatorname{Tr} \frac{1}{2} B X^{2}} e^{-\frac{1}{2} \bar{\phi} \phi}
$$

$X$ being a hermitian matrix, it can be diagonalized by a unitary transformation

$$
X=U x U^{-1} \quad x_{i j}=x_{i} \delta_{i j}
$$

Using this in (50) we find

$$
\begin{aligned}
\langle 2 p+1 \mid X, \phi\rangle & =(\sqrt{2 B})^{p N(N-1)}\left[\epsilon^{i_{1} \ldots i_{N}} \phi_{i_{1}}\left(U x U^{-1} \phi\right)_{i_{2}} \ldots\left(U x^{N-1} U^{-1} \phi\right)_{i_{N}}\right]^{2 p} e^{-\operatorname{Tr} \frac{1}{2} B X^{2}} e^{-\frac{1}{2} \bar{\phi} \phi} \\
& =(\sqrt{2 B})^{p N(N-1)}[\operatorname{det} U]^{2 p} \prod_{i<j}\left(x_{i}-x_{j}\right)^{2 p} \prod_{i=1}^{N}\left(U^{-1} \phi\right)_{i}^{2 p} e^{-\operatorname{Tr} \frac{1}{2} B X^{2}} e^{-\frac{1}{2} \bar{\phi} \phi}
\end{aligned}
$$

Using (52) we can express the scalar product of the $|2 p+1\rangle$ state as

$$
\begin{aligned}
\langle 2 p+1 \mid 2 p+1\rangle & =\int\left[d X_{i j}\right] d \bar{\phi}_{l} d \phi_{l}\langle 2 p+1 \mid X, \phi\rangle\langle X, \phi \mid 2 p+1\rangle \\
& \sim \int\left[d X_{i j}\right] \prod_{i<j}\left(x_{i}-x_{j}\right)^{4 p} e^{-\operatorname{Tr} B X^{2}} d \bar{\phi}_{l} d \phi_{l} \prod_{i}\left[\left(U^{-1} \phi\right)_{i}(\bar{\phi} U)_{i}\right]^{2 p} e^{-\bar{\phi} \phi}
\end{aligned}
$$

Since $U$ is a unitary matrix the integration over $\phi$ 's completely decouples (unlike the case in eq.(28)). Further 9]

$$
\left[d X_{i j}\right]=d x_{i} \prod_{i<j}\left(x_{i}-x_{j}\right)^{2}[d U]
$$

where $[d U]$ is the Haar measure. Integration over the nondiagonal elements of $X$ gives

$$
\langle 2 p+1 \mid 2 p+1\rangle \sim \int d x_{i} \prod_{i<j}\left(x_{i}-x_{j}\right)^{4 p+2} e^{-B \sum_{i} x_{i}^{2}}
$$


This is the probability distribution for the one-dimensional Calogero ground state wavefunction $\prod_{i<j}\left(x_{i}-x_{j}\right)^{2 p+1} e^{-B \sum_{i} x_{i}^{2} / 2}$. This is not surprising; it was already indicated in [2, 12] that the Chern-Simons matrix model is equivalent to the Calogero model.

Let us now use a coherent state representation for the Calogero wavefunction $\left\langle x_{1}, \ldots, x_{N} \mid \Psi\right\rangle=$ $\prod_{i<j}\left(x_{i}-x_{j}\right)^{2 p+1} e^{-B \sum_{i} x_{i}^{2} / 2}$. The coherent state representation of any wavefunction $\langle x \mid \Psi\rangle$ can be written as

$$
\langle z \mid \Psi\rangle=\int d x\langle z \mid x\rangle\langle x \mid \Psi\rangle
$$

where $\hat{z}|z\rangle=z|z\rangle$ and $\hat{z}=\sqrt{\frac{B}{2}}(\hat{x}+i \hat{y}), \quad[\hat{x}, \hat{y}]=\frac{i}{B}$. Using

$$
\langle z \mid x\rangle=e^{-\frac{B}{2} x^{2}} e^{\sqrt{2 B} \bar{z} x} e^{-\bar{z}^{2} / 2} e^{-|z|^{2} / 2}
$$

we find

$$
\langle z \mid \Psi\rangle=\int d x e^{-B\left(x-\frac{\bar{z}}{\sqrt{2 B}}\right)^{2}} f(x)
$$

where $f(x)$ is given by $\langle x \mid \Psi\rangle=f(x) e^{-B x^{2} / 2}$. In evaluating (58) we expand $f(x)$ around $\bar{z} / \sqrt{2 B}$.

$$
\begin{aligned}
\langle z \mid \Psi\rangle & =\left.e^{-\frac{|z|^{2}}{2}} \sum_{k=0}^{\infty} \int d x e^{-B\left(x-\frac{\bar{z}}{\sqrt{2 B}}\right)^{2}} \frac{1}{(2 k) !}\left(x-\frac{\bar{z}}{\sqrt{2 B}}\right)^{2 k} \frac{\partial^{2 k} f}{\partial x^{2 k}}\right|_{x=\bar{z} / \sqrt{2 B}} \\
& =\left.e^{-\frac{|z|^{2}}{2}} \sum_{k=0}^{\infty} \frac{\Gamma\left(k+\frac{1}{2}\right)}{(2 k) ! B^{k+\frac{1}{2}}} \frac{\partial^{2 k} f}{\partial x^{2 k}}\right|_{x=\bar{z} / \sqrt{2 B}} \\
& =\sqrt{\frac{\pi}{B}} e^{-\frac{|z|^{2}}{2}}\left[e^{\frac{1}{4 B} \frac{\partial^{2}}{\partial x^{2}}} f(x)\right]_{x=\bar{z} / \sqrt{2 B}}
\end{aligned}
$$

The coherent state representation of the many-body Calogero wavefunction is thus

$$
\left\langle z_{1}, \ldots, z_{N} \mid \Psi\right\rangle \sim e^{-\sum_{i}\left|z_{i}\right|^{2} / 2}\left[e^{\frac{1}{4 B} \sum_{i} \frac{\partial^{2}}{\partial x_{i}^{2}}} \prod_{i<j}\left(x_{i}-x_{j}\right)^{2 p+1}\right]_{x_{i}=\bar{z}_{i} / \sqrt{2 B}}
$$

For $p=0(60)$ gives the $\nu=1$ Laughlin state. For $p \neq 0$ the coherent representation of the Calogero state has a long distance behavior similar to the $\nu=\frac{1}{2 p+1}$ Laughlin state, but a different short distance behavior. This connection between the one-dimensional Calogero wavefunction and the Laughlin states has already been noted in 13 .

\section{Summary and discussion}

In an attempt to clarify the exact correspondence between the Chern-Simons matrix model introduced by Polychronakos and the fractional QHE at filling fraction $\nu=1 / m$, as described by Laughlin wavefunctions, we have derived the matrix model wavefunctions using a coherent state representation. We have presented two different coherent state representations, each one implementing a different choice for the phase space coordinates of the underlying one-dimensional fermionic system. 
In the $A$-representation, the eigenvalues of the matrix $A$ are identified with the phase space coordinates $z$ of the fermions, while in the $X$-representation, the eigenvalues of the matrix $X_{1}$ are identified with the one-dimensional coordinates $x$ of the fermions.

Both choices give identical results when $p=0$, or equivalently $\theta=0$ in (9). The corresponding wavefunction is identical to the $\nu=1$ Laughlin wavefunction.

For $p \neq 0$ the two representations give different results. Although the explicit expressions for the probability distributions are different, they share some common features. They both have the same long and short distance behavior. Comparing them to the corresponding $\nu=\frac{1}{2 p+1}$ Laughlin distributions, we find that it is only the long distance behavior which is in agreement. The short distance behavior does not agree with the Laughlin one.

A noticeable difference between the two representations is that the $X$-representation leads to a holomorphic wavefunction with antisymmetric properties, while this is not possible for the $A$-representation.

As we mentioned earlier there is an ambiguity in introducing electron coordinates in the matrix model. This has to do with the choice of coherent state representation. In this paper we analyzed two particular coherent state representations, which seem to be the natural choices from the matrix model point of view. In both cases we find that the emerging wavefunctions do not quite agree with the Laughlin one. Although this by itself does not prove that the original matrix model is not equivalent to the Laughlin theory for the $\nu=1 / m$ fractional QHE, it makes the precise correspondence between the two models less transparent. One can argue that there may exist another coherent state representation, corresponding to another choice for the electron coordinates, such that there is agreement with the Laughlin wavefunction. However, for the matrix model to be truly useful in the context of QHE, this new coordinate choice should be easily identifiable.

\section{Acknowledgements}

We would like to thank A.P. Polychronakos for many useful discussions on the matrix model and its relation to the Calogero model. Discussions with V.P. Nair are also acknowledged. This work was supported in part by the NSF grant PHY-9970724 and a PSC-31 CUNY award.

\section{References}

[1] L. Susskind, hep-th/0101029.

[2] A.P. Polychronakos, hep-th/0103013.

[3] S. Hellerman and M. Van Raamsdonk, hep-th/0103179. 
[4] A.P. Polychronakos, private communication.

[5] R.E. Prange and S.M. Girvin, The quantum Hall effect (Springer, Berlin, 1990); for a recent review see S. Girvin, cond-mat/9907002 and references therein.

[6] G. Dunne, R. Jackiw and C. Trugenberger, Phys. Rev. D41 (1990) 661; G. Dunne and R. Jackiw, Nucl. Phys. Proc. Suppl. 33C (1993) 114.

[7] S. Iso, D. Karabali and B. Sakita, Nucl. Phys. B388 (1992) 700; Phys. Lett. B296 (1992) 143.

[8] R. B. Laughlin, in The quantum Hall effect (Springer, Berlin, 1990); Phys. Rev. Lett. 50 (1983) 1395.

[9] E. Brezin, C. Itzykson, G. Parisi and J.B. Zuber, Comm. Math. Phys. 50 (1978) 35.

[10] J. Ginibre, JMP 6 (1964) 440; M. L. Mehta "Random Matrices and the Statistical Theory of Energy Levels", Academic Press 1967, New York.

[11] D. Callaway, Phys. Rev. B43 (1991) 8641.

[12] A.P. Polychronakos, Phys. Lett. B266 (1991) 29.

[13] H. Azuma and S. Iso, Phys. Lett. B331 (1994) 107. 